\title{
Contraction of cross-linked actomyosin bundles
}

\author{
Natsuhiko Yoshinaga ${ }^{1}$ and Philippe Marcq ${ }^{2}$ \\ ${ }^{1}$ WPI Advanced Institute for Materials Research (WPI-AIMR), Tohoku University, \\ Sendai 980-8577, Japan \\ ${ }^{2}$ Laboratoire Physico-Chimie Curie, Institut Curie, Université Pierre et Marie Curie, \\ CNRS UMR 168, 75005 Paris, France \\ E-mail: yoshinaga@wpi-aimr.tohoku.ac.jp, philippe.marcq@curie.fr
}

\begin{abstract}
Cross-linked actomyosin bundles retract when severed in vivo by laser ablation, or when isolated from the cell and micromanipulated in vitro in the presence of ATP. We identify the time scale for contraction as a viscoelastic time $\tau$, where the viscosity is due to (internal) protein friction. We obtain an estimate of the order of magnitude of the contraction time $\tau \approx 10-100 \mathrm{~s}$, consistent with available experimental data for circumferential microfilament bundles and stress fibers. Our results are supported by an exactly solvable, hydrodynamic model of a retracting bundle as a cylinder of isotropic, active matter, from which the order of magnitude of the active stress is estimated.
\end{abstract}

\section{Introduction}

Cells organise filamentous actin (F-actin) into complex cytoskeletal structures that play a major role in determining cell rheology and cell shape, and in generating the forces necessary for motility, cell division, or tissue stability [1]. Depending on the nature of the actin-binding proteins that nucleate, help polymerise, and cross-link actin filaments, the structure of F-actin networks varies widely [2, 3], from the near isotropic gels found in the cell cortex to the linear bundles that form filopodia or stereocilia [4, 5]. Passive cross-linkers, such as $\alpha$-actinin or fascin, contribute to the network elasticity, wheras bipolar mini-filaments of non-muscle myosin II motors that pull on neighbouring actin filaments of opposite polarity are responsible for contractility.

Cross-linked actomyosin bundles include the circumferential bundles (CBs) found near apical cell junctions of epithelia [6], and the stress fibers (SFs) assembled in the cytoskeleton of non-muscle animal cells that exert substantial traction forces on their environment [7]. They are robust and stable organelles, that can be extracted from the cell while maintaining their shape, structure, and contractility, over durations long enough to allow mechanical testing [8, 9, 10]. Indeed isolated circumferential bundles [9] as well as isolated stress fibers [10] contract in vitro in the presence of adenosine triphosphate (ATP). 
Laser photoablation stands out as a powerful and versatile tool, widely used to reveal the distribution, orientation, and relative intensity of stress in living cells and tissues (see [11, 12] for recent reviews). In order to investigate the cytoskeletal basis of the mechanical properties of cells and tissues, the retraction of ablated actomyosin bundles has been studied quantitatively by several groups [13, 14, 15, 16, 17]. These experiments give unambiguous evidence that the bundles are under tension in vivo, since they retract once severed, and that the retraction is an active process, since it is blocked by inhibitors of contractility [15, 16, 18]. The relaxation dynamics is generally fitted by a single exponential, that defines a contraction time, whereas the initial velocity at the time of ablation gives access to (relative) measurements of the preexisting bundle tension. Interestingly, the retraction that follows laser ablation is also relevant in (unperturbed) physiological conditions since stress fibers spontaneously rupture in vivo [19].

We use dimensional analysis to estimate the contraction time of actomyosin bundles, and show that the main source of dissipation during contraction is protein friction (section 2). The mechanical properties of retracting stress fibers have so far been interpreted with the help of models [17, 18, 20, 21] that emphasise the underlying sarcomeric structure. One exception is a discrete, numerical model based on the tensegrity hypothesis that accounts for much of the observed phenomenology [22]. In section 3, we introduce and solve a continuous model of a cross-linked actomyosin bundle, valid on hydrodynamic length scales large compared to the mesh size of the F-actin network, and where contractility is modeled by a constant active stress term [23, 24]. In section 4, comparison with experimental data yields the order of magnitude of the active stress of stress fibers and circumferential bundles.

\section{Contraction time}

The relaxation of the length of a stress fiber severed in vivo is well fitted by an exponential function of time, with a contraction time $\tau$ of the order of 1 to $10 \mathrm{~s}$, depending on cell type [16, 17, 18], and spatial position within the cell [25]. Laser ablation of the apical circumferential bundles of epithelial cells yields a somewhat longer contraction time of the order of 10 to $100 \mathrm{~s}$ [13, 14, 15, 26, 27]. Retracting bundles were first depicted as Kelvin-Voigt bodies [16], using a simple phenomenological model consisting in a dashpot and a spring in parallel, with a viscoelastic time equal to the ratio of the viscosity coefficient to the elastic modulus. However, the origin of the viscosity remains controversial. Internal viscosity dominates according to [17, whereas an external drag force is preferred in [20], implying a surprisingly large cytosolic viscosity of the order of $10 \mathrm{~Pa}$ s. In [22], the contraction time closely follows the (microscopic) viscoelastic time of components of the fiber, a parameter of the model.

In section 2.1, we estimate the orders of magnitude of the viscosity coefficient due to internal protein friction, of the elastic modulus of the bundle, and deduce the order of magnitude of the viscoelastic contraction time due to protein friction. In section 
2.2. using well established numerical values of the cytosolic viscosity, we further show that external viscous drag is negligible, and would lead to a much shorter viscoelastic time, at variance with experiment. We next evaluate the poroelastic time that governs the permeation of the cytosol through the F-actin network, and discuss its possible relevance to the dynamics of contraction. Finally, we calculate the order of magnitude of the elastic modulus and viscosity coefficient of stress fibers and circumferential bundles (section 2.3). An order of magnitude is hereafter defined by the integer value, obtained by truncation, of the decimal logarithm of the quantity of interest.

\subsection{Viscoelastic time}

We propose that the main source of dissipation in cross-linked actomyosin bundles lies in the binding-unbinding dynamics of cross-linkers on actin filaments. This dynamics leads to an effective (protein) friction, as introduced first in the context of rubber friction [28], and later for actomyosin dynamics in muscle cells [29].

In the linear regime, the protein friction force $\mathbf{F}_{p}$ exerted on one filament is proportional to its relative velocity $\mathbf{v}$ (with respect to neighbour filaments), with a friction coefficient $\zeta_{p}: \mathbf{F}_{p}=-\zeta_{p} \mathbf{v}$. Since the energy dissipated after unbinding was stored as elastic energy in strained cross-linkers, an estimate of the friction coefficient is 28]:

$$
\zeta_{p}=n_{X} k_{X} \tau_{X},
$$

where $n_{X}$ is the average number of attached cross-linkers per filament, $k_{X}$ is the spring constant of the cross-linker, and $\tau_{X}$ is the typical time for bond rupture. We expect that several cross-linker proteins, either passive or active, would contribute additively to the friction coefficient: $\zeta_{p}=\sum_{X} n_{X} k_{X} \tau_{X}$. To our knowledge, the spring constant of $\alpha$-actinin, arguably the most prevalent passive cross-linker in stress fibers, has not been measured. For lack of data, only protein friction due to myosin filaments will be taken into account. Using the numerical values $n_{X} \approx 10, k_{X} \approx 0.1 \mathrm{pN} \mathrm{nm}^{-1}$ for myosin filaments [30, 31], and equation (1), we obtain the order of magnitude of the microscopic friction coefficient $\zeta_{p} \approx 10^{-3}-10^{-2} \mathrm{~N} \mathrm{~s} \mathrm{~m}^{-1}$.

We expect the dissipative stress $\sigma_{p}$ due to protein friction to scale as $\sigma_{p} \approx n_{F} \zeta_{p} v / A$, where $n_{F}$ is the number of actin filaments in a section of the bundle, $A$ is the area of the section, and $v$ is the velocity. In three dimensions, protein friction translates into

a viscosity coefficient $\eta_{p}$, with $\sigma_{p}=\eta_{p} \frac{\partial v_{z}}{\partial z} \approx \eta_{p} \frac{U}{L}$, where $U$ and $L$ are the contraction velocity and the length of the bundle. Assuming for simplicity a constant velocity gradient $v_{z}=U \frac{z}{L}$, the local relative velocity on the scale of a filament is $v=U \frac{l_{F}}{L}$, where $l_{F}$ is the typical length of an actin filament (see figure 1 (B)). Note that although we have assumed that filaments are aligned in the $z$-direction, we may also consider an homogeneous, random orientation of filaments. This would modify the prefactors which arise from the average orientation of two filaments. Equating the two expressions for 
$\sigma_{p}$, we deduce the order of magnitude of the three-dimensional viscosity coefficient:

$$
\eta_{p} \approx n_{F} \frac{l_{F}}{A} \zeta_{p} .
$$

The stiffness of the bundle is dominated by cross-linkers, such as myosin filaments, known to be softer than actin filaments: $k_{X} \approx 0.1 \mathrm{pN} \mathrm{nm}^{-1}$ [30, 31] compared to $k_{F} \approx 10 \mathrm{pN} \mathrm{nm}^{-1}[32$, 33]. The elasticity of the bundle is characterised by a network structure of actin filaments and cross-linkers with long residual time. The network has two ends at $z=0$ and $z=L$ connected by various pathways in parallel. Each pathway has filaments and cross-linkers connected in series, and the bundle elasticity is approximated by that of a dominant pathway (figure 1 (C)). When hard and soft springs are connected in series, the force acting on the ensemble of two springs for the displacement $\Delta z$ is $F \simeq \Delta z /\left(k_{F}^{-1}+k_{X}^{-1}\right)$ where $k_{F}$ is a spring constant of a hard actin filament and $k_{X}$ is that of a soft cross-linker. When $k_{F} \gg k_{X}$, the force is dominated by $k_{X}$. The elastic stress $\sigma^{\mathrm{el}}$ is of order $\sigma^{\mathrm{el}}=E \frac{\partial u_{z}}{\partial z} \approx E \frac{\Delta Z}{L}$, where $E$ and $\Delta Z$ respectively denote the Young modulus, and the (macroscopic) displacement of the bundle. Denoting by $l_{X}$ the typical length between cross-linkers in the network, approximated by the length of a myosin filament, we expect the (microscopic) displacement of a cross-linker to scale as $\Delta z=\Delta Z \frac{l_{X}}{L}$. Since the number of cross-linkers in a section is $n_{X} n_{F}$, we find for the elastic stress $\sigma^{\mathrm{el}} \approx n_{X} n_{F} k_{X} \Delta z / A$. Since the two expressions for $\sigma^{\mathrm{el}}$ are equal, the order of magnitude of the Young modulus reads:

$$
E \approx n_{X} n_{F} \frac{l_{X}}{A} k_{X} .
$$

Using equations (17-3), we obtain the order of magnitude of the viscoelastic time due to protein friction:

$$
\tau=\frac{\eta_{p}}{E} \approx \frac{l_{F}}{l_{X}} \tau_{X},
$$

independent of the extension of the bundle. Indeed, experiments suggest that the contraction time is independent of the initial width $a_{0}$ [16], and of the initial length $l_{0}$ [34] of the ablated stress fiber, at odds with the prediction $\tau \propto l_{0}^{2}$ of the sarcomerebased model introduced in [20].

The typical lengths of actin and myosin filaments are respectively $l_{F} \approx 1 \mu \mathrm{m}$ and $l_{X} \approx 0.1 \mu \mathrm{m}$ [35, 36]. The association/dissociation rates of myosin light chain and $\alpha$-actinin have been measured in stress fibers in vivo in fluorescence recovery after photobleaching assays [37. The turn-over times are of order $\tau_{X} \approx 1-10 \mathrm{~s}$, consistent with the unbinding times measured on single actomyosin bonds under physiological loads [38, 39]. We obtain for the order of magnitude of the contraction time $\tau$ the range

$$
\tau \approx 10^{1}-10^{2} \mathrm{~s},
$$

in accord with experimental observations for contraction in vivo after laser ablation, as well as in vitro after extraction from the cell [9, 10]. 

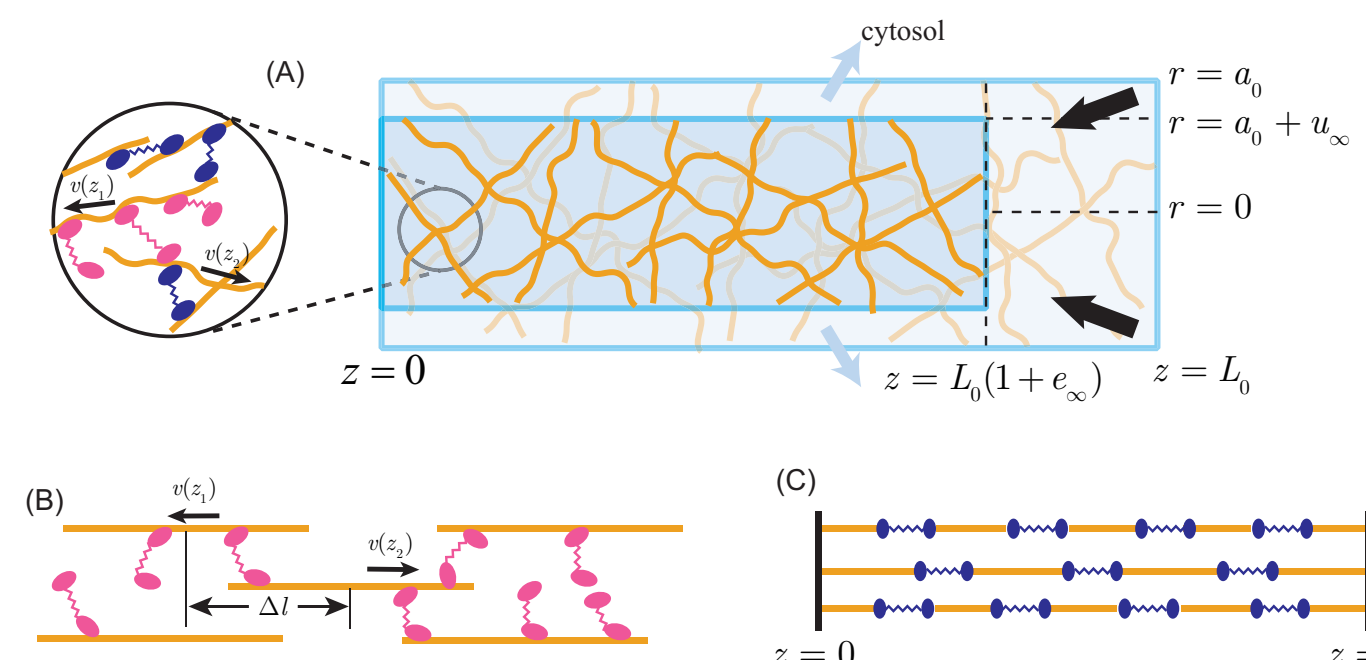

(C)

Figure 1. (Color Online) (A) Schematic image of the contraction of an actomyosin bundle. Permeating cytosol is squeezed out of the network during contraction. One end of the bundle is fixed at the origin $z=0$. The bundle contracts longitudinally from the initial length $L_{0}$ to the final length $L_{0}\left(1+e_{\infty}\right)\left(e_{\infty}<0\right)$, and radially from the initial radius $a_{o}$ to the final radius $a_{0}+u_{\infty}\left(u_{\infty}<0\right)$. The velocities of filaments whose center of mass positions are located at $z_{1}$ and $z_{2}$ are denoted as $v\left(z_{1}\right)=U z_{1} / L$ and $v\left(z_{2}\right)=U z_{2} / L$, respectively. Cross-linkers contributing to the bundle viscosity (resp. elasticity) are drawn in red (resp. blue). Importantly, the turn-over of both active motor filaments and passive cross-linking proteins gives rise to protein friction. The typical distance between the centers of mass of two filaments is $\Delta l \approx l_{F}$ as schematically shown in (B). The abstract network structure is sketched in (C) where many pathways connect the two ends $(z=0$ and $z=L)$; each pathway contains filaments and cross-linkers in series.

\subsection{Poroelastic time}

One obvious alternative source of dissipation is hydrodynamic friction, due to the viscosity of the cytosol. At small scale, the longitudinal friction coefficient due to viscous drag on an actin filament, modeled as a cylinder of length $l_{F}$ and diameter $d_{F}$, reads $\zeta_{D}=2 \pi \eta_{\text {cytosol }} l_{F} /\left(\ln \left(l_{F} / d_{F}\right)+\gamma_{/ /}\right)$, where $\eta_{\text {cytosol }}$ is the viscosity of the cytosol, and $\gamma_{/ /}$is a dimensionless number of order $10^{-1}$ [40]. Using $l_{F} \approx 1 \mu \mathrm{m}, d_{F} \approx 10 \mathrm{~nm}$, and well-established values for the cytosolic viscosity $\eta_{\text {cytosol }} \approx 10^{-3}-10^{-1} \mathrm{~Pa} \mathrm{~s}$ [41], we find $\zeta_{D} \approx 10^{-9}-10^{-7} \mathrm{~N} \mathrm{~s} \mathrm{~m}^{-1}$, negligible when compared to $\zeta_{p}$. At the scale of the bundle, with $l_{B} \approx 10 \mu \mathrm{m}, d_{B} \approx 0.1 \mu \mathrm{m}$, we obtain $\zeta_{D} \approx 10^{-8}-10^{-6} \mathrm{~N} \mathrm{~s} \mathrm{~m}^{-1} \ll \zeta_{p}$. Viscous drag against the cytosol may thus be neglected.

Further, cytosol is squeezed out of the biopolymer network during the contraction: permeation also contributes to the dissipation. The permeation of a solvent through an elastic network has been studied in the context of chemical gels (see [42] for a recent review of stress-diffusion coupling), and has led to interesting insights into, e.g., the mechanics of the poroelastic cytoskeleton [41]. In a two-component system where fluid cytosol (the solvent) permeates an elastic polymer matrix, the total stress is expressed as the sum of the elastic stress of the polymer network, and of a pressure term for the 
liquid solvent: $\sigma_{i j}^{\text {tot }}=\sigma_{i j}^{\mathrm{el}}-p \delta_{i j}$, where $\delta_{i j}$ is the identity tensor (see section 3 for a detailed calculation).

Supplementing this constitutive equation with Darcy's law for the permeation of the solvent, one obtains a diffusion equation for the displacement field of the gel, with a diffusion constant $D \simeq \kappa E$, where $\kappa$ is the permeability coefficient. Since $\kappa \propto \xi^{2} / \eta_{\text {cytosol }}$, where $\xi$ is the mesh size of the network and $\eta_{\text {cytosol }}$ is the cytosolic viscosity, the time scale for relaxation over a typical distance $a_{0}$, evaluated as the radius of the bundle, reads:

$$
\tau_{p}=\frac{a_{0}^{2}}{D} \approx\left(\frac{a_{0}}{\xi}\right)^{2} \frac{\eta_{\text {cytosol }}}{E} .
$$

From equation (3), and $A \approx a_{0}^{2}$, we expect the poroelastic time to be sensitive to the width of the bundle: $\tau_{p} \propto a_{0}^{4}$. The typical radius of a stress fiber is $a_{0}^{\mathrm{SF}} \approx 100$ $\mathrm{nm}$ [43], whereas the cross-sectional area of a circumferential bundle is of the order of $A^{\mathrm{CB}} \approx 1 \mu \mathrm{m}^{2}$ [8, 9, 13, 14], or $a_{0}^{\mathrm{CB}} \approx 1 \mu \mathrm{m}$. Using $\xi \approx 10 \mathrm{~nm}$ [8, 10] and $n_{F} \approx 10$ [10], we obtain:

$$
\begin{aligned}
\tau_{p}^{\mathrm{SF}} & \propto 10^{-6}-10^{-4} \mathrm{~s}, \\
\tau_{p}^{\mathrm{CB}} & \propto 10^{-2}-10^{0} \mathrm{~s} .
\end{aligned}
$$

Permeation of the cytosol through the polymer network occurs on diffusive times that are shorter than the observed contraction time, and may be safely ignored. It may however become relevant for very thick bundles: note that the upper bound of the estimate of the poroelastic time is only one order of magnitude shorter than observed contraction times in the case of circumferential bundles. In section 3.2, we introduce and solve a theoretical model of an actomyosin bundle as an active, viscoporoelastic material. We show in section 3.4 that the viscoelastic behavior, as discussed in section 2.1, is recovered in the limit where the viscoelastic time is much larger than poroelastic diffusion times.

\subsection{Material properties}

The Young modulus may be directly estimated using equation (3). For stress fibers, $A^{\mathrm{SF}} \approx 10^{-2} \mu \mathrm{m}^{2}$, whence $E^{\mathrm{SF}} \approx 10^{5} \mathrm{~Pa}$. In [43, 44], the force-extension curve of an isolated stress fiber has been measured in the absence of ATP, up to large deformations of the order of $200 \%$ where the fiber breaks. In the linear regime of small strains, the fiber's Young modulus was estimated to be of the order of $10^{5}$ to $10^{6} \mathrm{~Pa}$. Circumferential bundles are thicker $A^{\mathrm{CB}} \approx 1 \mu \mathrm{m}^{2}$, and correspondingly softer: we obtain from equation (3) $E^{\mathrm{CB}} \approx 10^{3} \mathrm{~Pa}$, somewhat higher than the value $E^{\mathrm{CB}} \approx 10^{2} \mathrm{~Pa}$ given in [45], where the elastic modulus of the actin cortex was measured in vivo in confluent endothelial cells.

Estimates of the bundle viscosity are obtained from equations (11) and (2). Since:

$$
\eta_{p} \approx n_{X} n_{F} \frac{l_{F}}{A} k_{X} \tau_{X}
$$


we find:

$$
\begin{aligned}
& \eta_{p}^{\mathrm{SF}} \approx 10^{6}-10^{7} \mathrm{~Pa} \mathrm{~s} \\
& \eta_{p}^{\mathrm{CB}} \approx 10^{4}-10^{5} \mathrm{~Pa} \mathrm{~s} .
\end{aligned}
$$

Although the effect of internal friction has been considered in models of stress fiber retraction [17, 20], this constitutes, to our knowledge, the first calculation of the numerical value of the viscosity coefficient. We emphasise that this value is an order of magnitude, derived from dimensional analysis, and defer a more rigorous treatment to future work.

\section{Models of active contraction}

We propose a three-dimensional, continuous, exactly solvable model of a cross-linked actomyosin bundle as an active, cylindrical body, inspired by active gel descriptions of the cytoskeleton [23, 24], where chemo-mechanical coupling is taken into account at linear order by including an active stress term in the constitutive equations. The resulting dynamical equations can be solved analytically, and yield results that agree well with experimental data. Related approaches successfully accounted for the possibility of oscillations in muscle sarcomeres, described as active elastic bodies [46, 47] and for the existence of distinct types of polarity patterns in contractile actomyosin bundles described as active, polar elastomers [48]. The assumptions made are discussed in detail in section 3.1. We introduce in section 3.2 a theoretical description of an actomyosin bundle as an active, viscoporoelastic material. We discuss in section 3.3 the poroelastic limit. In section 3.4, we show rigorously that the bundle behaves as an active viscoelastic body when the viscoelastic time is much larger than the poroelastic diffusion times, and solve the model in this limit.

\subsection{General formulation of the model}

We formulate a hydrodynamic description valid on length scales large compared to the typical mesh size of the underlying polymer network $d \gg \xi \approx 10 \mathrm{~nm}$ [8, 10]. In experiments, bundles are ablated far from their end points. We consider the retraction of one half of the severed bundle: the other tip is fixed and sets the origin of the $z$ axis. We consider the simplified geometry where (half-) a bundle initially adopts the shape of a cylinder of axis $O z$ (see figure $1(\mathrm{~A})$ ). We naturally use the cylindrical coordinates $(r, \theta, z)$. The bundle initially occupies the volume defined by $0 \leq r \leq a_{0}, 0 \leq z \leq l_{0}$, where $a_{0}$ and $l_{0}$ respectively denote the initial radius and length.

While this simplified geometry may seem a natural approximation for stress fibers spanning the ventral side of adherent cells and bound to the substrate at (fixed) focal adhesions, we believe that the following observations also justify its relevance for apical circumferential bundles. First, the ablation is generally performed along nearly straight

cell junctions: the local curvature may be neglected. Second, the shape of the cell is most often preserved despite the ablation of one cell junction, while other apical bundles in the 
same cell remain mostly unperturbed [13, 15], perhaps due to the stabilising influence of the surrounding epithelium. The retracting cell junction may therefore be treated as attached to an immobile cell-cell vertex and nearly independent of the remainder of the apical actin cortex.

The severed stress fibers do not retract when the ablation is performed in the presence of inhibitors of contractility [10, 16]. For this reason, the displacement field $\mathbf{u}$ is defined with respect to the initial state (immediately after ablation): $\mathbf{u}(t=0)=\mathbf{0}$. Following [49], we postulate the following functional form for components of $\mathbf{u}$ :

$$
\begin{aligned}
& u_{r}(r, \theta, z, t)=u(r, t) \\
& u_{\theta}(r, \theta, z, t)=0 \\
& u_{z}(r, \theta, z, t)=e(t) z .
\end{aligned}
$$

This Ansatz is central to our calculation. The displacement field is independent of $\theta$ due to rotational invariance about the axis $O z$. The origin is fixed, $u_{z}(z=0)=0$ at all times, and the longitudinal strain $e(t)$ is uniform. The initial cylindrical shape is preserved by our Ansatz for the displacement field, and the radius $a(t)$ and length $L(t)$ of the cylinder at a later time $t>0$ read:

$$
\begin{aligned}
& a(t)=a_{0}+u\left(a_{0}, t\right), \\
& L(t)=L_{0}(1+e(t)),
\end{aligned}
$$

where $e(t)$ appears as the relative variation of length of the bundle, negative for a contraction (see figure 1 1 (A)).

For simplicity, we assume that the bundle material properties are isotropic. In section 2, our estimates rely on the physical properties of cross-linkers, which remain approximately isotropic. In addition, the orientation of actin filaments inside actomyosin bundles shows a significant amount of disorder. The binding/unbinding kinetics of crosslinkers is taken into account by an effective, bulk viscous term with coefficient $\eta_{p}$, due to protein friction. We include in the constitutive equations a constant, isotropic, active stress $\sigma_{i j}^{\text {active }}=\sigma_{A} \delta_{i j}$, and $\sigma_{A}$ is positive since the bundle is under tension. The active stress $\sigma_{A}$ is assumed to be uniform, and independent of time: we neglect the banded myosin patterns that reflect the (disordered) sarcomeric organisation typical of stress fibers [50]. Note however that not all contractile actomyosin bundles exhibit a sarcomeric organisation [51, 52].

On the time scale involved, the final rest state is well defined. For stress fibers, the relative total retraction length $\left|e_{\infty}\right|$ is of the order of $10 \%$ [16, 17]: deformations are small enough for linear elasticity to hold. We denote by $E$ and $\nu$ the Young modulus and the Poisson ratio of the bundle, and by $K=\frac{E}{3(1-2 \nu)}$ and $G=\frac{E}{2(1+\nu)}$ the bulk and shear elastic moduli. Shear strains and shear elastic stresses cancel by construction. The normal elastic stresses read:

$$
\begin{aligned}
& \sigma_{r r}^{\mathrm{el}}=\frac{E}{(1+\nu)(1-2 \nu)}\left((1-\nu) \frac{\partial u}{\partial r}+\nu\left(\frac{u}{r}+e\right)\right), \\
& \sigma_{\theta \theta}^{\mathrm{el}}=\frac{E}{(1+\nu)(1-2 \nu)}\left((1-\nu) \frac{u}{r}+\nu\left(\frac{\partial u}{\partial r}+e\right)\right),
\end{aligned}
$$




$$
\sigma_{z z}^{\mathrm{el}}=\frac{E}{(1+\nu)(1-2 \nu)}\left((1-\nu) e+\nu\left(\frac{u}{r}+\frac{\partial u}{\partial r}\right)\right) .
$$

Like any organelle in a living cell, actomyosin bundles are highly dynamic structures: protein components constantly renew while the global organisation of the bundle is preserved. For stress fibers, fluorescence recovery assays performed in vivo yield an association/dissociation time of the order of a few minutes for actin [37, 53]. In this model, we assume that a steady-state is reached over the duration of ablation experiments, and accordingly that the material content of the bundle is constant during relaxation.

\subsection{Active viscoporoelastic contraction}

In this section, we study the permeation of a viscous liquid (the cytosol) through an active, elastic polymer network with transient cross-links. Active permeating gels have recently been studied in detail [54, 55]. Here, we follow a simpler approach, inspired by an exact calculation pertaining to the free swelling of a chemical gel whose initial shape is a cylinder [49], including two additional ingredients: a constant active stress, and a bulk viscous stress due to protein friction.

Since the strain field obeys the Ansatz (12,14), shear strains and shear elastic stresses cancel. The normal stresses read:

$$
\begin{aligned}
\sigma_{r r} & =\eta_{p} \frac{\partial v_{r}}{\partial r}+\sigma_{A}+\sigma_{r r}^{\mathrm{el}}, \\
\sigma_{\theta \theta} & =\eta_{p} \frac{v_{r}}{r}+\sigma_{A}+\sigma_{\theta \theta}^{\mathrm{el}}, \\
\sigma_{z z} & =\eta_{p} \frac{\partial v_{z}}{\partial z}+\sigma_{A}+\sigma_{z z}^{\mathrm{el}},
\end{aligned}
$$

where $\mathbf{v}=\frac{\partial \mathbf{u}}{\partial t}$ denotes the network velocity field. For a constant active stress $\sigma_{A}$, force balance reads:

$$
\begin{aligned}
& \partial_{r} p=\partial_{r} \sigma_{r r}^{\mathrm{el}}+\frac{1}{r}\left(\sigma_{r r}^{\mathrm{el}}-\sigma_{\theta \theta}^{\mathrm{el}}\right)+\eta_{p} \frac{\partial}{\partial r}\left(\frac{1}{r} \frac{\partial}{\partial r}\left(r v_{r}\right)\right), \\
& \partial_{\theta} p=\partial_{\theta} \sigma_{\theta \theta}^{\mathrm{el}}, \\
& \partial_{z} p=\partial_{z} \sigma_{z z}^{\mathrm{el}},
\end{aligned}
$$

where $p$ is the pressure field of the cytosol. Since $\partial_{\theta} \sigma_{\theta \theta}^{\mathrm{el}}=\partial_{z} \sigma_{z z}^{\mathrm{el}}=0$, we have $\partial_{\theta} p=\partial_{z} p=0$ : the pressure field is a function of radius and time only, $p=p(r, t)$. The radial dependence of the pressure field is obtained from equation (23) after integration with respect to $r$, using the boundary condition $p(a, t)=p_{\text {ext }}$, where $p_{\text {ext }}$ denotes the hydrodynamic pressure in the surrounding cytosol. We find:

$$
p(r, t)=p_{\mathrm{ext}}+\eta_{p}\left[\frac{1}{r} \frac{\partial}{\partial r}\left(r v_{r}\right)\right]_{a}^{r}+\frac{(1-\nu) E}{(1+\nu)(1-2 \nu)}\left[\frac{1}{r} \frac{\partial}{\partial r}(r u)\right]_{a}^{r} .
$$

Using the previous equation, we eliminate the pressure field from Darcy's law $\vec{\nabla}$. $\vec{v}=\kappa \nabla^{2} p$, where $\kappa$ is the permeability, and obtain the following equation for the 
displacement field:

$$
\frac{\partial u}{\partial t}+\frac{1}{2} \frac{\mathrm{d} e}{\mathrm{~d} t} r=D \frac{\partial}{\partial r}\left(\frac{1}{r} \frac{\partial}{\partial r}(r u)\right)+l_{p}^{2} \frac{\partial}{\partial r}\left(\frac{1}{r} \frac{\partial}{\partial r}\left(r v_{r}\right)\right),
$$

where $l_{p}^{2}=\kappa \eta_{p}, D=\frac{1-\nu}{(1+\nu)(1-2 \nu)} \kappa E$ is a diffusion coefficient, and their ratio

$$
\tau_{E}=\frac{l_{p}^{2}}{D}=\frac{(1+\nu)(1-2 \nu)}{1-\nu} \frac{\eta_{p}}{E}
$$

is a viscoelastic time.

The boundary condition at $r=a, \sigma_{r r}(r=a, t)=-p_{\text {ext }}$, yields:

$$
\frac{E}{(1+\nu)(1-2 \nu)}\left((1-\nu){\frac{\partial u}{\partial r}{ }_{\mid a}}+\nu\left(\frac{u(a)}{a}+e\right)\right)+\eta_{p}{\frac{\partial v_{r}}{\partial r}{ }_{\mid a}}_{a}=-\sigma_{A} .
$$

In the case of free contraction, the external force applied on the tip of the cylinder at $z=L$ is equal to $\int_{0}^{a} \mathrm{~d} r 2 \pi r \sigma_{z z}(r, t)=-\pi a^{2} p_{\text {ext }}$. Combining the two boundary conditions, we obtain:

$$
\tau_{G} \frac{\mathrm{d}}{\mathrm{d} t}\left(\frac{u(a)}{a}-e\right)=-\left(\frac{u(a)}{a}-e\right),
$$

where $\tau_{G}$ is a viscoelastic time based on the shear elastic modulus:

$$
\tau_{G}=\frac{\eta_{p}}{2 G}
$$

which contributes to the relaxation provided that $\frac{u\left(a_{0}, t=0\right)}{a_{0}} \neq e(t=0)$, see also equations (A.12 A.13). Here, the initial strain is equal to zero $u\left(a_{0}, t=0\right)=e(t=0)=0$, and the solution of equation (30) is for all time $t$ :

$$
e(t)=\frac{u(a, t)}{a} \simeq \frac{u\left(a_{0}, t\right)}{a_{0}}
$$

The length and the radius of the bundle both relax in time according to the same functional form since $e(t)=\frac{L(t)-L_{0}}{L_{0}}=\frac{a(t)-a_{0}}{a_{0}}$.

Equations (27,29,32) are solved by the expansion:

$$
\begin{aligned}
& e(t)=e_{\infty}+\sum_{n} e_{n} e^{-t / \tau_{n}}, \\
& u(r, t)=u_{\infty}(r)+\sum_{n} u_{n}(r) e^{-t / \tau_{n}},
\end{aligned}
$$

where the $\tau_{n}$ are relaxation times. Equation (27) yields a differential equation obeyed by the amplitudes $u_{n}$ and $e_{n}$ :

$$
u_{n}+\frac{1}{2} e_{n} r=D\left(\frac{\tau_{E}-\tau_{n}}{\tau_{n}}\right) \frac{\mathrm{d}}{\mathrm{d} r}\left(\frac{1}{r} \frac{\mathrm{d}}{\mathrm{d} r}\left(r u_{n}\right)\right) .
$$

In the following, we define the lengths $l_{n}$ by $l_{n}^{2}=D\left|\tau_{E}-\tau_{n}\right|$.

In the long time limit, the stationary solution reads:

$$
\begin{aligned}
& e_{\infty}=-\frac{\sigma_{A}}{3 K}, \\
& u_{\infty}(r)=-\frac{\sigma_{A}}{3 K} r .
\end{aligned}
$$


Remarkably, we predict that actomyosin bundles also contract along the radial direction $\left(a_{\infty}<a_{0}\right)$, independently of the value of the Poisson ratio $\nu$, since the final radius reads:

$$
a_{\infty}=a_{0}\left(1+e_{\infty}\right)=a_{0}\left(1-\frac{\sigma_{A}}{3 K}\right) .
$$

Indeed, electron microscopy imaging indicates that isolated stress fibers contract radially [10. To our knowledge, this observation has not been confirmed by optical imaging in a live cell, due to insufficient spatial resolution.

The relaxational dynamics are solved as follows. When $\tau_{E}>\tau_{n}$, the functions $u_{n}(r)$ are the solutions of an inhomogeneous, modified Bessel equation:

$$
u_{n}(r)=b_{n} I_{1}\left(\frac{r}{l_{n}}\right)-\frac{1}{2} e_{n} r
$$

where the coefficient $b_{n}$ is an amplitude, and $I_{1}(x)$ is a modified Bessel function of the first kind. Since $u_{n}\left(a_{0}\right)=a_{0} e_{n}$ (see equation (32) ), we obtain $e_{n}=\frac{2 b_{n}}{3 a_{0}} I_{1}\left(\frac{a_{0}}{l_{n}}\right)$ from equation (39). We deduce from equation (29) that the relaxation times $\tau_{n}$ are solutions of the equation:

$$
\left(\frac{\tau_{E}}{\tau_{n}}-1\right)\left[\frac{a_{0}}{l_{n}} I_{1}^{\prime}\left(\frac{a_{0}}{l_{n}}\right)-\frac{1}{3} I_{1}\left(\frac{a_{0}}{l_{n}}\right)\right]=\frac{4}{3} \frac{\nu}{1-\nu} I_{1}\left(\frac{a_{0}}{l_{n}}\right) .
$$

When $\tau_{n}>\tau_{E}$, equation (35) is an inhomogeneous Bessel equation. Modified Bessel functions are replaced by Bessel functions:

$$
u_{n}(r)=b_{n} J_{1}\left(\frac{r}{l_{n}}\right)-\frac{1}{2} e_{n} r
$$

where $J_{1}(x)$ is a Bessel function of the first kind, and $e_{n}=\frac{2 b_{n}}{3 a_{0}} J_{1}\left(\frac{a_{0}}{l_{n}}\right)$. The relaxation times $\tau_{n}$ are solutions of the equation:

$$
\left(\frac{\tau_{E}}{\tau_{n}}-1\right)\left[\frac{a_{0}}{l_{n}} J_{1}^{\prime}\left(\frac{a_{0}}{l_{n}}\right)-\frac{1}{3} J_{1}\left(\frac{a_{0}}{l_{n}}\right)\right]=\frac{4}{3} \frac{\nu}{1-\nu} J_{1}\left(\frac{a_{0}}{l_{n}}\right),
$$

which may be obtained from equation (40) upon replacing $I_{1}$ by $J_{1}$. In figure 2, we plot as a function of the parameter $\frac{\tau_{E}}{\tau_{p}}$ the dimensionless relaxation times $\frac{\tau_{n}}{\tau_{p}}$, at a given value of the Poisson ratio. Equation (40) admits only one solution, whereas equation (42) admits an infinity of solutions, which contribute to the expansions (33) 34).

\subsection{Active poroelastic limit}

In the limit where $\tau_{n} \gg \tau_{E}$, equation (42) becomes:

$$
\frac{a_{0}}{r_{n}} J_{1}^{\prime}\left(\frac{a_{0}}{r_{n}}\right)+\frac{5 \nu-1}{3(1-\nu)} J_{1}\left(\frac{a_{0}}{r_{n}}\right)=0,
$$

with $r_{n}=\sqrt{D \tau_{n}}$, in agreement with [49]. The largest solution of equation (43) is $\tau_{n} \approx \tau_{p}=\frac{a_{0}^{2}}{D}$ : this limit is realised when $\tau_{p} \gg \tau_{E}$, as may be possible, e.g., for very thick bundles assembled in vitro, since we expect the poroelastic time to scale as the fourth power of bundle radius, see equation (6) and below. 


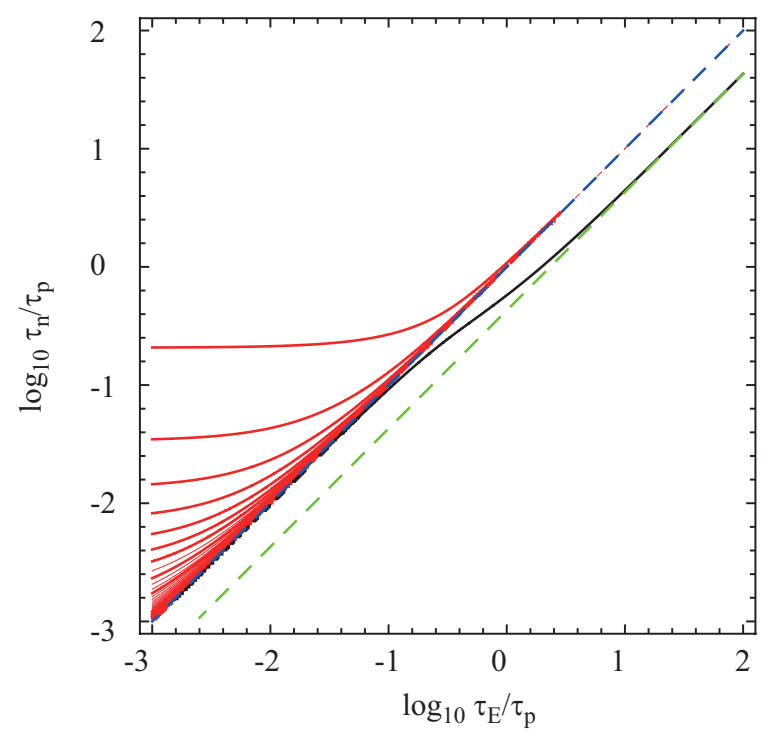

Figure 2. (Color Online) Dimensionless relaxation times $\frac{\tau_{n}}{\tau_{p}}$ as a function of the parameter $\frac{\tau_{E}}{\tau_{p}}$, obtained numerically for $\nu=0.4$. The dashed blue line $\left(\frac{\tau_{n}}{\tau_{p}}=\frac{\tau_{E}}{\tau_{p}}\right)$ separates the two sets of solutions. The black line gives the unique solution of equation (40), which converges to $\frac{\tau_{K}}{\tau_{p}}$ as $\frac{\tau_{E}}{\tau_{p}} \rightarrow \infty$ (active viscoelastic limit, dashed green line). The red lines are the solutions of equation (42), and converge to constant values as $\frac{\tau_{E}}{\tau_{p}} \rightarrow 0$ (active poroelastic limit, solutions of equation (43)).

\subsection{Active viscoelastic limit}

We formally define the viscoelastic limit as the regime where the permeability coefficient $\kappa$ diverges. Darcy's law then implies that the cytosolic pressure field is homogeneous $(\nabla p=0)$. Since $a_{0} / l_{n} \propto 1 / \sqrt{\kappa}$, we may use $x I_{1}^{\prime}(x) \simeq I_{1}(x)$ and $x J_{1}^{\prime}(x) \simeq J_{1}(x)$ in equations (40) and (42) since $0<x \ll 1$. For both equations, we obtain a unique solution $\tau_{n} \rightarrow \tau_{K}$ :

$$
\tau_{K}=\frac{\eta_{p}}{3 K}
$$

a viscoelastic time based on the bulk modulus $K$. The expansions (33) 34 reduce to one exponential term. Given the initial conditions $u(r, t=0)=e(t=0)=0$, we find the expression of the displacement fields:

$$
\begin{aligned}
& u_{r}(r, t)=-\frac{\sigma_{A}}{3 K} r\left(1-e^{-t / \tau_{K}}\right), \\
& u_{z}(z, t)=-\frac{\sigma_{A}}{3 K} z\left(1-e^{-t / \tau_{K}}\right) .
\end{aligned}
$$

The length of the ablated bundle decreases as an exponential function of time, as observed in experiments. Equation (46) also yields the initial retraction velocity $v_{0}$, proportional to the active stress:

$$
v_{0}=v_{z}\left(z=L_{0}, t=0\right)=-L_{0} \frac{\sigma_{A}}{\eta_{p}} .
$$

We may rewrite the argument $a_{0} / l_{n}$ as a function of time scales: $\frac{a_{0}}{l_{n}}=\sqrt{\frac{\tau_{p}}{\left|\tau_{E}-\tau_{n}\right|}}$. We find that $\tau_{E}-\tau_{K}=\frac{2 \nu}{1-\nu} \tau_{K}: a_{0} \ll l_{n}$ corresponds to $\tau_{n} \rightarrow \tau_{K} \approx \tau_{E}-\tau_{K} \gg \tau_{p}$. The 
contraction of an active viscoporoelastic cylinder (with protein friction) does indeed reduce to an active viscoelastic behavior, provided that the viscoelastic time is large compared to the poroelastic time. The cross-over between the two regimes occurs when $\tau_{p} \approx \tau_{K}$, or $a_{0}^{2} \approx \kappa \eta_{p}$ : it may be controlled by the bundle radius, with a cross-over radius $a_{c}$. Using $\kappa \approx \xi^{2} / \eta_{\text {cytosol }}$ and $\eta_{p} \approx n_{F} \frac{l_{F}}{a_{c}^{2}} \zeta_{p}$ (equation (2) ), we find:

$$
a_{c} \approx\left(\frac{n_{F} l_{F} \zeta_{p} \xi^{2}}{\eta_{\text {cytosol }}}\right)^{1 / 4} .
$$

Using the same numbers as in section 2, we obtain the value $a_{c} \approx 10 \mu \mathrm{m}$, large compared to the radius of actomyosin bundles found in cells. The separation of time scale $\tau_{K} \gg \tau_{p}$ no longer exists when $a_{0} \gg a_{c}$, and is absent without protein friction, i.e. when crosslinkers are fixed and do not unbind over the time scale considered.

In Appendix A, we solve a model of an actomyosin bundle as an active, viscoelastic material, and check that the results are identical to the limit presented in this section. In addition, we consider the case where the initial state differs from the reference state, and find that the strain, initially nonzero, relaxes as a linear combination of two exponentials with characteristic times $\tau_{K}$ and $\tau_{G}$, equations (A.12 A.13).

\section{Active stress}

In experiments, the stationary longitudinal strain is of order $\left|e_{\infty}\right| \approx 10^{-1}$. Assuming a value of the Poisson ratio of order $\nu=0.4$ [56, [57], the estimates of section 2 remain relevant since $3 K \approx E$. Using equation (36), we deduce the order of magnitude of the active stress $\sigma_{A}=3 K\left|e_{\infty}\right| \approx E\left|e_{\infty}\right|$ :

$$
\begin{aligned}
& \sigma_{A}^{\mathrm{SF}} \approx 10^{4} \mathrm{~Pa}, \\
& \sigma_{A}^{\mathrm{CB}} \approx 10^{2} \mathrm{~Pa} .
\end{aligned}
$$

Since the stall force of a myosin motor is of order $F_{S} \approx 1 \mathrm{pN}$ [58], the above values agree with a naive estimate of the active stress as

$$
\sigma_{A} \approx n_{X}^{A} n_{F} \frac{F_{S}}{A}
$$

where $n_{X}^{A}$ is the number of active myosin molecules (per filament) that generate stress, with the same order of magnitude as the total number of attached cross-linkers per filament $n_{X}^{A} \approx n_{X}$. Since $L_{0}-L_{\infty}=\left|e_{\infty}\right| L_{0}$, combining equations (3) and (51) yields:

$$
L_{0}-L_{\infty} \approx \frac{F_{S}}{k_{X} l_{X}} L_{0} .
$$

Experiments suggest that the total retracted length is independent of the bundle radius $a_{0}[16]$.

The value of the active stress obtained for circumferential bundles is close to the order of magnitude obtained for cortical actin $\sigma_{A}^{\mathrm{CA}} \approx 10^{3} \mathrm{~Pa}$ [59]. Estimate (49) suggests that cells whose stress fibers pull on an area of the order of $1 \mu \mathrm{m}^{2}$ exert locally a force of the order $F_{A} \approx 10 \mathrm{nN}$. This is consistent with measurements of traction forces exerted 
by cells on micropatterned pillars [60]. Adherent cells assemble thicker and more robust stress fibers when the rigidity of the substrate is large [61]. In this case traction forces saturate to a value independent of the external stiffness. In [62], a simple model of the cytoskeleton as an active, elastic material allowed to identify active contractility as the physical origin of the saturation traction force, yielding $10^{4} \mathrm{~Pa}$ as an order of magnitude of the active stress, in agreement with (49).

The values of $e_{\infty}$ measured in ablation assays are similar to the values of the "preexisting strain" measured in isolated stress fibers [43], or of the "preextension" upon unloading the stress fibers of cells adhering on a stretchable substrate [63]. Indeed, as we have seen in (36 37), actomyosin contraction may be seen as equivalent to a reduction in the stress-free reference length of an elastic material [64]. Experiments show that the amplitude of the preextension correlates positively with contractility [63], in qualitative agreement with equations (36-37). Further, knock-down of $\alpha$-actinin increases preextension [63]. Given that stress fibers depleted in $\alpha$-actinin are expected to be softer [65], this observation also fits with equations (36) [37), since a smaller value of the elastic modulus at fixed active stress leads to a larger strain.

\section{Conclusion}

Local ablation is a widely used tool to estimate the value of the local stress in living cells, up to a (generally unknown) viscosity coefficient (see equations (47) and (A.14)). Ascribing the physical origin of the fiber's viscosity to protein friction, itself due to the association/dissociation dynamics of cross-linkers, we obtain the order of magnitude of the viscosity coefficient $\eta_{p}$. Using an independent estimate of the bundle's elastic modulus, we deduce the order of magnitude of the contraction time $\tau \approx 10-100 \mathrm{~s}$, interpreted as a viscoelastic time. We find that the order of magnitude of the poroelastic time scales is much smaller, and study rigorously the limit where poroelasticity may be neglected thanks to an exactly solvable model of the contracting bundle. We identify a well-defined threshold, controlled for instance by the bundle width: viscoelasticity (resp. poroelasticity) dominates when the viscoelastic time is much longer (resp. much shorter) than the poroelastic time. For an isotropic material, the model predicts that contraction occurs both in the longitudinal and radial directions, irrespective of the value of the Poisson ratio. From the observed longitudinal strain, we deduce the order of magnitude of the active stress: $\sigma_{A}^{\mathrm{CB}} \approx 10^{2} \mathrm{~Pa}$ in circumferential bundles and $\sigma_{A}^{\mathrm{SF}} \approx 10^{4} \mathrm{~Pa}$ in stress fibers. Following the literature, we distinguish stress fibers from circumferential bundles. However their protein constituents and macroscopic properties are identical: the differences we emphasise may in fact be superficial, and turn out to conceal a continuum of parameter values.

We expect our results to be relevant to the mechanics of other cytoskeletal structures, such as the actin purse string at the circumference of healing wounds [66], or the supra-cellular actin cable that surrounds the amnioserosa during dorsal closure of fly embryos [67]. Circular, contractile actomyosin bundles also form in adherent (single) 
fibroblasts during the course of spreading [68]. Modeling a retracting bundle as a straight cylinder is relevant locally along a ring as long as the retracted length is small compared to the radius. It may be interesting to generalise the calculation presented here from a cylindrical to a toroidal geometry. Strikingly, ablation experiments performed at the tissue level in Drosophila pupal epithelia, on the scale of one hundred cells, reveal a relaxation time of the same order as the contraction time of individual circumferential bundles [69], suggesting that the same physical mechanism may be at play.

We strongly believe that estimates of orders of magnitude, based on dimensional analysis, are a useful contribution to quantitative biology [70]. More quantitative data will be needed in order to reduce the spread of numerical values. One promising direction in which our ideas may be tested is that of bundles reconstituted in vitro [71, 72, 73, 74], where microscopic and macroscopic parameters may be varied in a controlled fashion. For instance, we expect that the contraction time increases linearly with the average length of actin filaments $l_{F}$ (equation (4)), a prediction that may be tested experimentally. On the theoretical side, one would like to be able to calculate in a rigorous manner the material parameters of cross-linked actomyosin bundles from the microscopic parameters pertaining to individual constituents (filaments and crosslinkers). This important question is left for future study.

\section{Acknowledgments}

The authors thank Axel Buguin, Damien Cuvelier, François Graner, Shinji Deguchi, Jean-François Joanny, Jacques Prost and Tetsuo Yamaguchi for fruitful discussions, as well as anonymous referees for constructive criticism. N. Y. acknowledges support by a Grant-in-Aid for Young Scientists (B) (No.23740317). P. M. would like to thank Prof. Masaki Sano and Prof. Takao Ohta for their kind hospitality, as well as the Kavli Institute for Theoretical Physics, with partial support by the National Science Foundation under Grant No. NSF PHY05-51164. This work was supported by JSPS, MAEE and MESR under the Japan-France Integrated Action Program (SAKURA).

\section{Appendix A. Active viscoelastic contraction}

Using the same Ansatz for the displacement field, equations (12, 14), the constitutive equations for the active, viscoelastic contraction of a cylinder of isotropic material read:

$$
\begin{aligned}
& \sigma_{r r}=\eta_{p} \frac{\partial v_{r}}{\partial r}+\sigma_{A}+\frac{E}{(1+\nu)(1-2 \nu)}\left((1-\nu) \frac{\partial u}{\partial r}+\nu\left(\frac{u}{r}+e\right)\right), \\
& \sigma_{\theta \theta}=\eta_{p} \frac{v_{r}}{r}+\sigma_{A}+\frac{E}{(1+\nu)(1-2 \nu)}\left((1-\nu) \frac{u}{r}+\nu\left(\frac{\partial u}{\partial r}+e\right)\right), \\
& \sigma_{z z}=\eta_{p} \frac{\mathrm{d} e}{\mathrm{~d} t}+\sigma_{A}+\frac{E}{(1+\nu)(1-2 \nu)}\left((1-\nu) e+\nu\left(\frac{u}{r}+\frac{\partial u}{\partial r}\right)\right) .
\end{aligned}
$$

Again, since $u$ and $e$ are independent of $\theta$ and $z$, the azimuthal and longitudinal components of the force balance equation are immediately verified. In the radial 
direction, we find:

$$
\eta_{p} \partial_{r}\left(\frac{1}{r} \partial_{r}\left(r v_{r}\right)\right)+\frac{E(1-\nu)}{(1+\nu)(1-2 \nu)} \partial_{r}\left(\frac{1}{r} \partial_{r}(r u)\right)=0 .
$$

A radial displacement linear in $r$ :

$$
u(r, t)=\frac{1}{2} A(t) r
$$

solves equation (A.4), provided that the dimensionless function $A(t)$ depends on time only. The stress field is then uniform (with $\sigma_{\theta \theta}=\sigma_{r r}$ ):

$$
\begin{aligned}
\sigma_{r r} & =\frac{1}{2} \eta_{p} \frac{\mathrm{d} A}{\mathrm{~d} t}+\sigma_{A}+\frac{E}{(1+\nu)(1-2 \nu)}\left((1-\nu) \frac{A}{2}+\nu\left(\frac{A}{2}+e\right)\right), \\
\sigma_{z z} & =\eta_{p} \frac{\mathrm{d} e}{\mathrm{~d} t}+\sigma_{A}+\frac{E}{(1+\nu)(1-2 \nu)}((1-\nu) e+\nu A),
\end{aligned}
$$

and determined by the boundary conditions.

Neglecting the external, cytosolic pressure, normal stresses at the free surface are $\sigma_{n n \mid r=a}=\sigma_{n n \mid z=L}=0$. We obtain a system of two coupled differential equations for $A(t)$ and $e(t)$ :

$$
\begin{aligned}
& (1+\nu)(1-2 \nu) \frac{\eta_{p}}{E} \frac{\mathrm{d} A}{\mathrm{~d} t}=-A-2 \nu e-2(1+\nu)(1-2 \nu) \frac{\sigma_{A}}{E} \\
& (1+\nu)(1-2 \nu) \frac{\eta_{p}}{E} \frac{\mathrm{d} e}{\mathrm{~d} t}=-\nu A+(\nu-1) e+(1+\nu)(1-2 \nu) \frac{\sigma_{A}}{E}
\end{aligned}
$$

diagonalised as:

$$
\begin{aligned}
\tau_{K} \frac{\mathrm{d}}{\mathrm{d} t}(A+e) & =-(A+e)-\frac{\sigma_{A}}{K}, \\
\tau_{G} \frac{\mathrm{d}}{\mathrm{d} t}(2 e-A) & =-(2 e-A),
\end{aligned}
$$

where $\tau_{G}$ and $\tau_{K}$ are defined in equations (31) and (44) respectively. For zero initial displacements, we find $A(t)=2 e(t)$, and $e(t)=-\frac{\sigma_{A}}{3 K}\left(1-e^{-t / \tau_{K}}\right)$, in agreement with equations (45,46).

If we now take into account a possible difference between the initial state and the stress-free reference state of the passive bundle, the initial conditions become $A(t=0)=A_{0} \neq 0, e(t=0)=e_{0} \neq 0$. The strain relaxes as a linear combination of two exponentials with characteristic times $\tau_{G}$ and $\tau_{K}$ :

$$
\begin{aligned}
A(t)=-\frac{2 \sigma_{A}}{3 K}\left(1-e^{-t / \tau_{K}}\right) & +\frac{2}{3}\left(A_{0}+e_{0}\right) e^{-t / \tau_{K}} \\
& +\frac{1}{3}\left(A_{0}-2 e_{0}\right) e^{-t / \tau_{G}}, \\
e(t)=-\frac{\sigma_{A}}{3 K}\left(1-e^{-t / \tau_{K}}\right) & +\frac{1}{3}\left(A_{0}+e_{0}\right) e^{-t / \tau_{K}} \\
& -\frac{1}{3}\left(A_{0}-2 e_{0}\right) e^{-t / \tau_{G}} .
\end{aligned}
$$

A nonzero initial elastic stress also contributes to the recoil velocity:

$$
v_{0}=-L_{0} \frac{\sigma_{A}+\sigma_{z z}^{\mathrm{el}}(t=0)}{\eta_{p}},
$$


with $\sigma_{z z}^{\mathrm{el}}(t=0)=\frac{E}{(1+\nu)(1-2 \nu)}\left((1-\nu) e_{0}+\nu A_{0}\right)$. Since $\frac{\tau_{K}}{\tau_{G}}=\frac{1-2 \nu}{1+\nu}$, the active contraction of bundles initially stretched or compressed may yield a measurement of the Poisson ratio. In [14], the relaxation of ablated circumferential bundles has been fitted by a sum of two exponential functions with relaxation times $\tau_{1}$ and $\tau_{2}$, with $\frac{\tau_{1}}{\tau_{2}} \approx 10$. Assuming that our model is valid, this observation suggests that, in this experiment, $\frac{\tau_{K}}{\tau_{G}} \approx 10$, or equivalently $\frac{1}{2}-\nu \approx 10^{-1}$.

For completeness, we also solve the case of an incompressible material. The dynamical equations for $A(t)$ and $e(t)$ read:

$$
\begin{gathered}
\tau_{G} \frac{\mathrm{d} A}{\mathrm{~d} t}+A=-\frac{\sigma_{A}}{G}, \\
\tau_{G} \frac{\mathrm{d} e}{\mathrm{~d} t}+e=-\frac{\sigma_{A}}{2 G} .
\end{gathered}
$$

We find that the bundle contracts both radially and longitudinally with a characteristic time $\tau_{G}$, the viscoelastic time based on the shear elastic modulus:

$$
\begin{aligned}
& A(t)=-\frac{\sigma_{A}}{G}+\left(\frac{\sigma_{A}}{G}+A_{0}\right) e^{-t / \tau_{G}}, \\
& e(t)=-\frac{\sigma_{A}}{2 G}+\left(\frac{\sigma_{A}}{2 G}+e_{0}\right) e^{-t / \tau_{G}} .
\end{aligned}
$$

\section{References}

[1] T D Pollard, W C Earnshaw, and J Lippincott-Schwartz. Cell Biology. Saunders, 2007.

[2] J Stricker, T Falzone, and M L Gardel. Mechanics of the F-actin cytoskeleton. J. Biomech., 43:9-14, 2009.

[3] O Lieleg, M Claessens, and A R Bausch. Structure and dynamics of cross-linked actin networks. Soft Matter, 6:218 - 225, 2010.

[4] R Furukawa and M Fechheimer. The structure, function, and assembly of actin filament bundles. Int. Rev. Cytol., 175:29-90, 1997.

[5] J R Bartles. Parallel actin bundles and their multiple actin-bundling proteins. Curr. Op. Cell Biol., 12:72-78, 2000.

[6] M Cavey and T Lecuit. Molecular bases of cell-cell junctions stability and dynamics. Cold Spring Harb. Perspect. Biol., 1:a002998, 2009.

[7] S Pellegrin and H Mellor. Actin stress fibres. J. Cell Sci., 120:3491-3499, 2007.

[8] K Owaribe, R Kodama, and G Eguchi. Demonstration of contractility of circumferential actin bundles and its morphogenetic significance in pigmented epithelium in vitro and in vivo. J. Cell Biol., 90:507-514, 1981.

[9] K Owaribe and H Masuda. Isolation and characterization of circumferential microfilament bundles from retinal pigmented epithelial cells. J. Cell Biol., 95:310-315, 1982.

[10] K Katoh, Y Kano, M Masuda, H Onishi, and K Fujiwara. Isolation and contraction of the stress fiber. Mol. Biol. Cell, 9:1919-1938, 1998.

[11] J Colombelli, E G Reynaud, and E H K Stelzer. Investigating relaxation processes in cells and developing organisms: from cell ablation to cytoskeleton nanosurgery. Methods Cell Biol., 82:267-291, 2007.

[12] M Rauzi and P-F Lenne. Cortical forces in cell shape changes and tissue morphogenesis. Curr. Top. Dev. Biol., 95:93-144, 2011.

[13] M Rauzi, P Verant, T Lecuit, and P-F Lenne. Nature and anisotropy of cortical forces orienting Drosophila tissue morphogenesis. Nat. Cell Biol., 10:1401-1410, 2008. 
[14] K P Landsberg, R Farhadifar, J Ranft, D Umetsu, T J Widmann, T Bittig, A Said, F Jülicher, and C Dahmann. Increased cell bond tension governs cell sorting at the Drosophila anteroposterior compartment boundary. Curr. Biol., 19:1950-1955, 2009.

[15] R Fernandez-Gonzalez, S de Matos Simoes, J-C Röper, S Eaton, and J A Zallen. Myosin II dynamics are regulated by tension in intercalating cells. Dev. Cell, 17:736-743, 2009.

[16] S Kumar, I Z Maxwell, A Heisterkamp, T R Polte, T P Lele, M Salanga, E Mazur, and D E Ingber. Viscoelastic retraction of single living stress fibers and its impact on cell shape, cytoskeletal organization, and extracellular matrix mechanics. Biophys. J., 90:3762-3773, 2006.

[17] J Colombelli, A Besser, H Kress, E G Reynaud, P Girard, E Caussinus, U Haselmann, J V Small, U S Schwarz, and E H K Stelzer. Mechanosensing in actin stress fibers revealed by a close correlation between force and protein localization. J. Cell Sci., 122:1665-1679, 2009.

[18] R J Russell, S-L Xia, R B Dickinson, and T P Lele. Sarcomere mechanics in capillary endothelial cells. Biophys. J., 97:1578-1585, 2009.

[19] M A Smith, E Blankman, M L Gardel, L Luettjohann, C M Waterman, and M C Beckerle. A zyxin-mediated mechanism for actin stress fiber maintenance and repair. Dev. Cell, 19:365-376, 2010.

[20] M R Stachowiak and B O'Shaughnessy. Recoil after severing reveals stress fiber contraction mechanisms. Biophys. J., 97:462-471, 2009.

[21] A Besser, J Colombelli, E H K Stelzer, and U S Schwarz. Viscoelastic response of contractile filament bundles. Phys. Rev. E, 83:051902, 2011.

[22] Y Luo, X Xu, T Lele, S Kumar, and D E Ingber. A multi-modular tensegrity model of an actin stress fiber. J. Biomech., 41:2379-2387, 2008.

[23] K Kruse, J-F Joanny, F Jülicher, J Prost, and K Sekimoto. Generic theory of active polar gels: a paradigm for cytoskeletal dynamics. Eur. Phys. J. E, 16:5-16, 2005.

[24] F Jülicher, K Kruse, J Prost, and J-F Joanny. Active behavior of the cytoskeleton. Phys. Rep., 449:3-28, 2007.

[25] K Tanner, A Boudreau, M J Bissell, and S Kumar. Dissecting regional variations in stress fiber mechanics in living cells with laser nanosurgery. Biophys. J., 99:2775-2783, 2010.

[26] R Farhadifar, J-C Röper, B Aigouy, S Eaton, and F Jülicher. The influence of cell mechanics, cell-cell interactions, and proliferation on epithelial packing. Curr. Biol., 17:2095-2104, 2007.

[27] M Cavey, M Rauzi, P-F Lenne, and T Lecuit. A two-tiered mechanism for stabilization and immobilization of E-cadherin. Nature, 453:751-756, 2008.

[28] A Schallamach. A theory of dynamic rubber friction. Wear, 6:375-382, 1963.

[29] K Tawada and K Sekimoto. A physical model of ATP-induced actin-myosin movement in vitro. Biophys. J., 59:343-356, 1991.

[30] T Neumann, M Fauver, and G H Pollack. Elastic properties of isolated thick filaments measured by nanofabricated cantilevers. Biophys. J., 75:938-947, 1998.

[31] C Veigel, M L Bartoo, D C White, J C Sparrow, and J E Molloy. The stiffness of rabbit skeletal actomyosin cross-bridges determined with an optical tweezers transducer. Biophys. J., 75:1424$1438,1998$.

[32] H Kojima, A Ishijima, and T Yanagida. Direct measurement of stiffness of single actin filaments with and without tropomyosin by in vitro nanomanipulation. P.N.A.S., 91:12962-12966, 1994.

[33] X Liu and G H Pollack. Mechanics of F-actin characterized with microfabricated cantilevers. Biophys. J., 83:2705-2715, 2002.

[34] J Colombelli. Private communication.

[35] G Langanger, M Moeremans, G Daneels, A Sobieszek, M De Brabander, and J De Mey. The molecular organization of myosin in stress fibers of cultured cells. J. Cell Biol., 102:200-209, 1986.

[36] T M Svitkina, I G Surguchova, A B Verkhovsky, V I Gelfand, M Moeremans, and J De Mey. Direct visualization of bipolar myosin filaments in stress fibers of cultured fibroblasts. Cell Motil. Cytoskel., 12:150-156, 1989. 
[37] $\mathrm{P}$ Hotulainen and $\mathrm{P}$ Lappalainen. Stress fibers are generated by two distinct actin assembly mechanisms in motile cells. J. Cell Biol., 173:383-394, 2006.

[38] T Nishizaka, R Seo, H Tadakuma, K Kinosita, and S Ishiwata. Characterization of single actomyosin rigor bonds: load dependence of lifetime and mechanical properties. Biophys. J., 79:962-974, 2000.

[39] J M Ferrer, H Lee, J Chen, B Pelz, F Nakamura, R D Kamm, and M J Lang. Measuring molecular rupture forces between single actin filaments and actin-binding proteins. Proc. Natl Acad. Sci. U.S.A., 105:9221-9226, 2008.

[40] M M Tirado and J García de la Torre. Translational friction coefficients of rigid, symmetric top macromolecules. Application to circular cylinders. J. Chem. Phys., 71:2581, 1979.

[41] G T Charras, T J Mitchison, and L Mahadevan. Animal cell hydraulics. J. Cell Sci., 122:3233$3241,2009$.

[42] M Doi. Gel dynamics. J. Phys. Soc. Japan, 78:052001, 2009.

[43] S Deguchi, T Ohashi, and M Sato. Evaluation of tension in actin bundle of endothelial cells based on preexisting strain and tensile properties measurements. Mol. Cell Biomech., 2:125-133, 2005.

[44] S Deguchi, T Ohashi, and M Sato. Tensile properties of single stress fibers isolated from cultured vascular smooth muscle cells. J. Biomech., 39:2603-2610, 2006.

[45] W Feneberg, M Äpfelbacher, and E Sackmann. Microviscoelasticity of the apical cell surface of human umbilical vein endothelial cells (HUVEC) within confluent monolayers. Biophys. J., $87: 1338-1350,2004$.

[46] S Günther and K Kruse. Spontaneous waves in muscle fibres. New J. Phys., 9:417, 2007.

[47] S Banerjee and M C Marchetti. Instabilities and oscillations in isotropic active gels. Soft Matter, 7:463-473, 2011.

[48] N Yoshinaga, J-F Joanny, J Prost, and P Marcq. Polarity patterns of stress fibers. Phys. Rev. Lett., 105:238103, 2010.

[49] T Yamaue and M Doi. The stress diffusion coupling in the swelling dynamics of cylindrical gels. J. Chem. Phys., 122:084703, 2005.

[50] L J Peterson, Z Rajfur, A S Maddox, C D Freel, Y Chen, M Edlund, C O, and K Burridge. Simultaneous stretching and contraction of stress fibers in vivo. Mol. Biol. Cell, 15:3497-3508, 2004.

[51] L P Cramer, M Siebert, and T J Mitchison. Identification of novel graded polarity actin filament bundles in locomoting heart fibroblasts: implications for the generation of motile force. J. Cell Biol., 136:1287-1305, 1997.

[52] T Mseka, M Coughlin, and L P Cramer. Graded actin filament polarity is the organization of oriented actomyosin ii filament bundles required for fibroblast polarization. Cell Motil Cytoskeleton, 66:743 - 753, 2009.

[53] J J Campbell and M M Knight. An improved confocal FRAP technique for the measurement of long-term actin dynamics in individual stress fibers. Microscopy Res. Tech., 70:1034-1040, 2007.

[54] J F Joanny, F Jülicher, K Kruse, and J Prost. Hydrodynamic theory for multi-component active polar gels. New J. Phys., 9:422, 2007.

[55] A C Callan-Jones and F Jülicher. Hydrodynamics of active permeating gels. New J. Phys., 13:093027, 2011.

[56] F G Schmidt, F Ziemann, and E Sackmann. Shear field mapping in actin networks by using magnetic tweezers. Eur Biophys J, 24:348-353, 1996.

[57] W R Trickey, F P T Baaijens, T A Laursen, L G Alexopoulos, and F Guilak. Determination of the Poisson's ratio of the cell: recovery properties of chondrocytes after release from complete micropipette aspiration. J Biomech, 39:78-87, 2006.

[58] J Howard. Mechanics of Motor Proteins and the Cytoskeleton. Sinauer Associates, 2005.

[59] G Salbreux, J Prost, and J-F Joanny. Hydrodynamics of cellular cortical flows and the formation of contractile rings. Phys. Rev. Lett., 103:058102, 2009. 
[60] J L Tan, J Tien, D M Pirone, D S Gray, K Bhadriraju, and C S Chen. Cells lying on a bed of microneedles: an approach to isolate mechanical force. Proc. Natl Acad. Sci. U.S.A., 100:14841489, 2003.

[61] M Ghibaudo, A Saez, L Trichet, A Xayaphoummine, J Browaeys, P Silberzan, A Buguin, and B Ladoux. Traction forces and rigidity sensing regulate cell functions. Soft Matter, 4:1836 $1843,2008$.

[62] P Marcq, N Yoshinaga, and J Prost. Rigidity sensing explained by active matter theory. Biophys. J., 101:L33-L35, 2011.

[63] L Lu, Y Feng, W J Hucker, S J Oswald, G D Longmore, and F C-P Yin. Actin stress fiber pre-extension in human aortic endothelial cells. Cell Motil. Cytoskel., 65:281-294, 2008.

[64] S Deguchi, T S Matsui, and K Iio. The position and size of individual focal adhesions are determined by intracellular stress-dependent positive regulation. Cytoskeleton, 68:639-651, 2011.

[65] Y Tseng and D Wirtz. Mechanics and multiple-particle tracking microheterogeneity of alphaactinin-cross-linked actin filament networks. Biophys. J., 81:1643-1656, 2001.

[66] M Tamada, T D Perez, W J Nelson, and M P Sheetz. Two distinct modes of myosin assembly and dynamics during epithelial wound closure. J. Cell Biol., 176:27-33, 2007.

[67] A Rodriguez-Diaz, Y Toyama, D L Abravanel, J M Wiemann, A R Wells, U S Tulu, G S Edwards, and D P Kiehart. Actomyosin purse strings: renewable resources that make morphogenesis robust and resilient. HSFP J., 2:220-237, 2008.

[68] Y Senju and H Miyata. The role of actomyosin contractility in the formation and dynamics of actin bundles during fibroblast spreading. J. Biochem., 145:137-150, 2009.

[69] I Bonnet, P Marcq, F Bosveld, L Fetler, Y Bellaïche, and F Graner. Mechanical state, material properties and continuous description of an epithelial tissue. J Roy Soc Lond Interface, 2012, doi: 10.1098/rsif.2012.0263.

[70] R Phillips and R Milo. A feeling for the numbers in biology. Proc. Natl Acad. Sci. U.S.A., 106:21465-21471, 2009.

[71] D Vignjevic, D Yarar, M D Welch, J Peloquin, T Svitkina, and G G Borisy. Formation of filopodialike bundles in vitro from a dendritic network. J. Cell Biol., 160:951-962, 2003.

[72] M M A E Claessens, M Bathe, E Frey, and A R Bausch. Actin-binding proteins sensitively mediate F-actin bundle stiffness. Nat. Mat., 5:748 - 753, 2006.

[73] D Strehle, J Schnauss, C Heussinger, J Alvarado, M Bathe, J Käs, and B Gentry. Transiently crosslinked F-actin bundles. Eur. Biophys. J., 40:93-101, 2011.

[74] T Thoresen, M Lenz, and M L Gardel. Reconstitution of contractile actomyosin bundles. Biophys. J., 100:2698-2705, 2011. 